http://jmscr.igmpublication.org/home/

ISSN (e)-2347-176x ISSN (p) 2455-0450

crossref DOI: https://dx.doi.org/10.18535/jmscr/v8i2.164

\title{
Vulval Filariasis: A Case Report
}

Authors

\section{Pushpendra Kanaujia ${ }^{1}$, Ashfaque Khan ${ }^{2}$, Abhishek Kulkarni ${ }^{3}$}

${ }^{1}$ Assistant Professor Department of General Surgery, GSVM Medical College, Kanpur

${ }^{2}$ Junior Resident-3 Department of General Surgery, GSVM Medical College, Kanpur

${ }^{3}$ Junior Resident-2 Department of General Surgery, GSVM Medical College, Kanpur

*Corresponding Author

\section{Ashfaque Khan}

Junior Resident-3 Department of General Surgery, GSVM Medical College, Kanpur, UP, India

\begin{abstract}
Introduction: Lymphoedema is defined as accumulation of lymph in the soft tissue due to obstruction of lymphatics resulting in accumulation of protein rich interstitial fluid. This accumulated lymph activates fibroblasts and mast cells leading to organization of oedema fluid and sclerosing fibrosis of subcutaneous tissue which gives rise to firm, non pitting and irreversible swelling associated with hyperkeratosis, verrucous and condylomatous changes. These features of long-standing lymph stasis are collectively called as Elephantiasis. Here we present a rare case of vulval filariasis in a 14-year-old unmarried female.

Case Presentation: Miss. XXX, a 14-year-old unmarried female, resident of Babupurva, Kanpur, presented to the OPD, Department of General Surgery, L.L.R hospital, Kanpur with complaints of growth over vulva for the past 11 years. The swelling which was initially the size of a pea was first noticed by her mother when she was 3 years old and assumed it to be a boil. The swelling gradually increased in size. However, the patient neglected it. Patient gave history that the swelling rapidly increased in size over the past three years to attain the present size. General examination and systemic examination was normal. The patient attained menarche 2 years back. Local examination revealed a $9 \times 7 \mathrm{~cm}$ boss elated elliptical pendulous mass projecting between the labia obstructing the vulval cleft. The skin over the mass was hard, thickened, hyperpigmented and had extensive rugosities. The mass was also non-pitting and non-tender.

Conclusion: Asvulval elephantiasis is a very rare entity today, we have compiled this case report to present the findings collected and shine light on the treatment protocols of a very rare diagnosis.
\end{abstract}

\section{Introduction}

Lymphoedema is defined as accumulation of lymph in the soft tissue due to obstruction of lymphatics resulting in accumulation of protein rich interstitial fluid. This accumulated lymph activates fibroblasts and mast cells leading to organization of oedema fluid and sclerosing fibrosis of subcutaneous tissue which gives rise to firm, non pitting and irreversible swelling associated with hyperkeratosis, verrucous and condylomatous changes. These features of longstanding lymph stasis are collectively called as Elephantiasis. The term Elephantiasis was first described by Celsius (30 BC-50 AD). Greek terminology esthioneme is used to describe elephantiasis which means to eat and carries an idea as something is gnawed, eroded or ulcerated. Lymphoedema can be primary or secondary. Congenital and or inherited condition causing accumulation of lymph is referred to as primary 
lymph oedema and if it is caused with pathological condition, it is called as secondary lymphoedema. Secondary causes of vulval lymphedema may be filarial, tubercular, chlamydial infection, lymphogranuloma venereum, post vulvectomy, postradiotherapy, after inguinal and pelvic lymph node dissection or idiopathic. Other causes include oncocerca volvulus, loaloa and mansonella perstans and rarely metastatic crohn's disease. Commonest infective filariasis results with Wuchereria Bancrofti and Brugia Malayi. 90\% of cases in humans is caused by Wuchereria bancrofti. Vulval Elephantiasis is extremely rare and accounts with 1-2 \% of total cases of genital Elephantiasis. Though rare, it is associated with significant physical disability and mental depression. In majority of the reported cases, bilateral labia are involved. Here we present a rare case of vulval filariasis in a 14-year-old unmarried female.

\section{Case Presentation}

Miss. XXX, a 14-year-old unmarried female, resident of Babupurva, Kanpur, presented to the OPD, Department of General Surgery, L.L.R hospital, Kanpur with complaints of growth over vulva for the past 11 years. The swelling which was initially the size of a pea was first noticed by her mother when she was 3 years old and assumed it to be a boil. The swelling gradually increased in size. However, the patient neglected it. Patient gave history that the swelling rapidly increased in size over the past three years to attain the present size. General examination and systemic examination was normal. The patient attained menarche 2 years back. Local examination revealed a $9 * 7 \mathrm{~cm}$ boss elated elliptical pendulous mass projecting between the labia obstructing the vulval cleft. The skin over the mass was hard, thickened, hyperpigmented and had extensive rugosities. The mass was also non-pitting and non-tender.

Routine blood investigations, chest $\mathrm{x}$ ray and ultrasound abdomen were unremarkable. Mantoux test was negative. The patient was treated with diethylcarbamazine (DEC) in three divided doses of $6 \mathrm{mg} / \mathrm{kg} /$ day for 21 days and was advised to wear tight undergarments and to maintain local hygiene to prevent any secondary infection. The patient was posted for surgery after the completion of course of DEC.

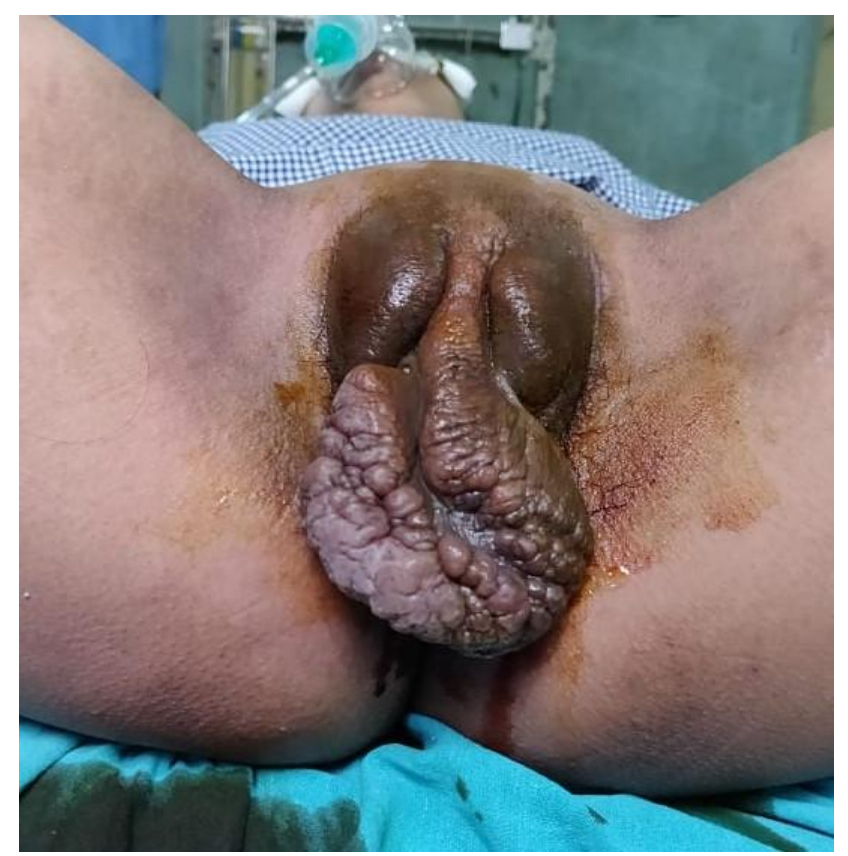

Figure 1: Pre- Operative Photograph of the Filarial Mass Covering the Vulval Cleft

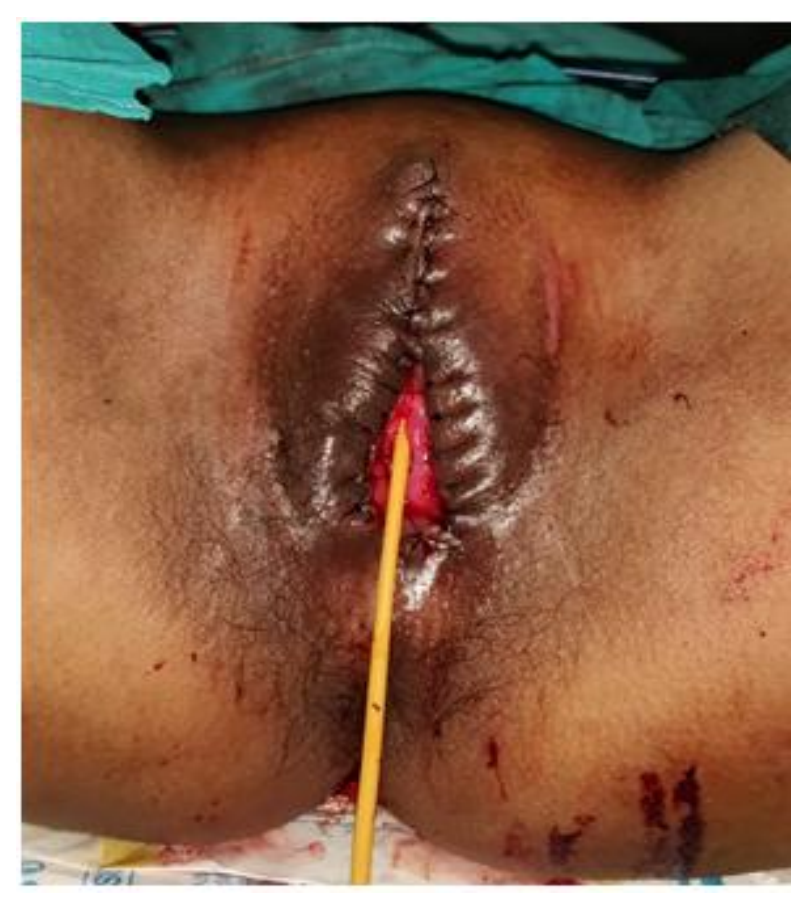

Figure 2: Immediete Post- Operative Photograph after Excision of the mass and Vulvoplasty 


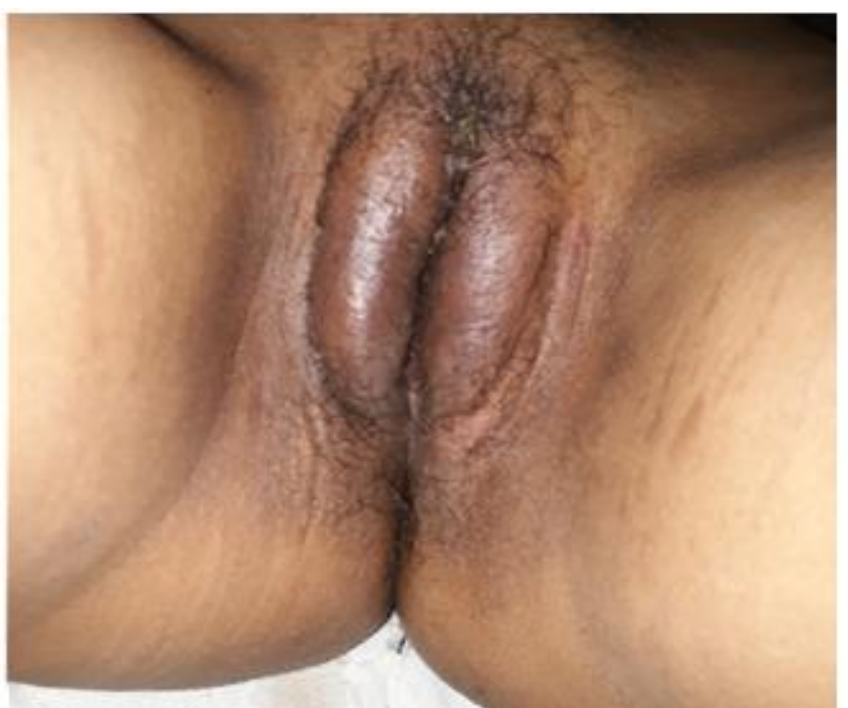

Figure 3: Follow Up Photograph of the Patient taken after 2 Weeks

\section{Discussion}

Lymphatic filariasis is caused mainly by Wuchereria bancrofti and Brugiamalai. Mosquito bite paves entry for third-stage microfilarias into the human body. The microfilariae quickly migrate towards the lymphatic system and begin to grow into adult forms. The migration of the adult worm through the lymphatics causes lymphatic damage mainly by producing inflammatory reactions and lymphangitis leading to stasis of lymph and subsequent lymphedema. To overcome the stasis, affected lymphatics dilate, become tortuous and a number of collateral lymph vessels develop leading to hypertrophy and hyperplasia of lymphatic endothelium further aggravating stasis. It is postulated that as long as the adult worms are alive, the lymphatic vessels remain patent. Death of the worm, however, leads to extensive peri lymphangitis, progressive fibrosis and obliteration of vessels. Therefore, the worm is usually not found in peripheral blood or within the involved tissue in these patients and hence clinical suspicion along with histopathological examination are the cornerstones of diagnosis.

Filarial lymphedema is treated with Diethylcarbamazine (DEC) in three divided doses of $6 \mathrm{mg} / \mathrm{kg} /$ day for 21 days which kills adults and as well as microfilariae. Leg elevation, elastic stocking, decongestive physiotherapy and maintenance of good skin care is also recommended. Surgical treatments includes excision of skin and subcutaneous tissue of involved part and primary closure of defect with normal adjacent skin. Partial thickness graft or rotation flap can be used if raw area is large. Surgical treatment is used only in extreme cases in order to reduce the weight of the affected organ, to help minimize the frequency of inflammatory attacks, to improve cosmesis, and to potentially reduce the risk of secondary angiosarcoma.

In our case, the patient came to us with a large vulval swelling with complaints of difficulty in walking and carrying out her day to day chores. The surgery was carried out to relieve her of the symptoms, improve cosmesis and alleviate her fears of the mass creating problems to her future marital life.

\section{Conclusion}

As vulval elephantiasis is a very rare entity today, we have compiled this case report to present the findings collected and shine light on the treatment protocols of a very rare diagnosis.

\section{Consent}

Written consent was obtained from patient and one of her relative regarding publication of finding and associated images

\section{References}

1. Mohan H, Bisht B, Goel P, et al. Vulval elephantiasis: a case report. Case Rep Infect Dis 2012;2012:1-3.

2. Satish Kumar Ranjan, Mini Sinha, Nawal K Jha1, D K Sinha, Amrisha Sharan, Giant Vulvar Elephantiasis of Filarial Origin: A Rare Case Report, IOSR Journal of Dental and Medical Sciences (IOSRJDMS), Volume 14, Issue 11 Ver. II (Nov. 2015), PP 38-40

3. Dr. Tanushree Mondal, Dr. Koushik Dewan, Dr. Partha Mandal, Case Report Of A Rare Case Of Vulval Elephantiasis, 
IOSR Journal of Dental and Medical Sciences (IOSR-JDMS), Volume 14, Issue 4 Ver. X (Apr. 2015), PP 101-102

4. Chakraborty M, Banu H, Chakraborty PP. BMJ Case Rep Published Online First: [please include Day Month Year]. doi:10.1136/ bcr-2018-224250

5. Dr. Sonali Deshmukh, Dr. D. K. Soyam, Dr. Madhuri Kilnake, Dr. R. Koreti, Vulval Filariasis - A Case Report, International Journal of Science and Research (IJSR), Volume 8 Issue 3, March 2019. 